This is an electronic reprint of the original article. This reprint may differ from the original in pagination and typographic detail.

Author(s): Morozov, Dmitry; Groenhof, Gerrit

Title: $\quad$ Hydrogen Bond Fluctuations Control Photochromism in a Reversibly Photo-Switchable Fluorescent Protein

Year: $\quad 2016$

Version:

Please cite the original version:

Morozov, D., \& Groenhof, G. (2016). Hydrogen Bond Fluctuations Control

Photochromism in a Reversibly Photo-Switchable Fluorescent Protein. Angewandte Chemie International Edition, 55(2), 576-578.

https://doi.org/10.1002/anie.201508452

All material supplied via JYX is protected by copyright and other intellectual property rights, and duplication or sale of all or part of any of the repository collections is not permitted, except that material may be duplicated by you for your research use or educational purposes in electronic or print form. You must obtain permission for any other use. Electronic or print copies may not be offered, whether for sale or otherwise to anyone who is not an authorised user. 


\title{
Hydrogen bond fluctuations control photochromism in a reversibly photo-switchable fluorescent protein
}

\author{
Dmitry Morozov, ${ }^{[\mathrm{a}]}$ and Gerrit Groenhof ${ }^{*[a]}$
}

\begin{abstract}
Reversibly switchable fluorescent proteins (RSFPs) are essential for high-resolution microscopy of biological samples, but the reason why these proteins are photochromic is still poorly understood. To address this problem we have performed molecular dynamics simulations of the fast switching Met159Thr mutant of the RSFP Dronpa. Our simulations revealed a ground state structural heterogeneity in the chromophore pocket that consists of three populations with one, two or three hydrogen bonds to the phenolate moiety of the chromophore. By means of non-adiabatic QM/MM molecular dynamics simulations, we demonstrated that the subpopulation with a single hydrogen bond is responsible for offswitching through photo-isomerization of the chromophore, whereas two or more hydrogen bonds inhibit the isomerization and promote fluorescence instead. While rational design of new RSFPs has so far focused on structure alone, our results suggest that structural heterogeneity must be considered as well.
\end{abstract}

Fluorescence microscopy with fluorescent proteins is playing an ever-increasing role not only in revealing how living systems function, but also in understanding diseases and finding new strategies to fight them. ${ }^{1}$ Recent developments have even pushed the spatial resolution of this technique beyond the diffraction limit. ${ }^{2}$ One strategy for achieving nanometer resolution in cells is to use reversibly photo-switchable fluorescent proteins (RSFPs), ${ }^{3}$ whose fluorescence can be turned on and off repeatedly with different wavelengths. However, achieving the full potential of this technique for high resolution in vivo imaging requires significant improvements of these photochromic protein labels.

Proteins of the photochromic Dronpa family have promising switching characteristics, ${ }^{4}$ but the achievable resolution is limited due to a low photostability. What further limits their application in fluorescence nanoscopy is that photo-switching and fluorescence occur at the same wavelength. Although transient spectroscopy, ${ }^{5}$ x-ray crystallography ${ }^{6}$ and $\mathrm{NMR}^{7}$ have provided important information about the switching, these insights have so far not been sufficient to overcome the limitations of RSFPs through rational protein design. A notable exception is Dreiklang that has different wavelengths for fluorescence and switching, ${ }^{8}$ but the blue-shift into the UV limits its use in vivo.

The main obstacle of structure-based optimization approaches is

[a] Dr. M. Morozov, Dr. G. Groenhof

Nanoscience Center \& Department of Chemistry

University of Jyväskylä

P. O. Box 35 40014, Jyväskylä, Finland

E-mail: gerrit.x.groenhof@jyu.fi

Supporting information for this article is given via a link at the end of the document. that the photo-switching is essentially a dynamic process Optimizing the switching therefore requires a complete understanding of the underlying molecular dynamics and the influence of the protein environment. As the relevant time and spatial resolution are notoriously hard to access by experiment, we have used hybrid quantum mechanics/molecular mechanics (QM/MM) simulations to reveal that photochromicity is controlled by structural fluctuations that have so far not been considered in the protein optimization process.
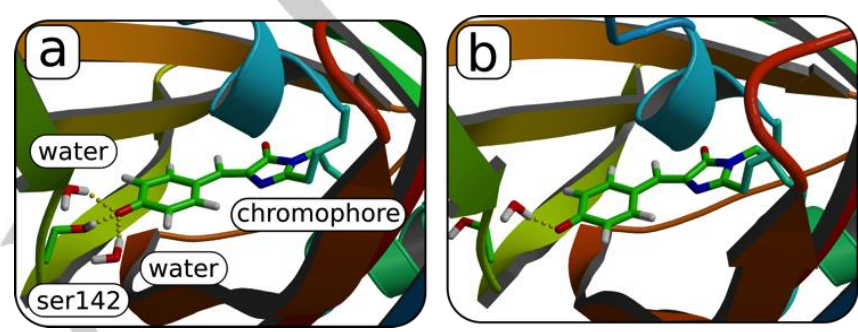

Figure 1. Close up of chromophore pocket in the M159T mutant of Dronpa, with an intact (a) and a transiently broken hydrogen bond network (b).

Because the low quantum yield of photo-switching in Dronpa would require far too many trajectories for a systematic investigation of the switching mechanism, we performed our simulations on the M159T mutant instead (Figure 1), ${ }^{9}$ which has a much higher quantum yield of off-switching. ${ }^{10}$ Replacing the bulky methionine by a threonine increases the volume around the chromophore. ${ }^{9}$ In previous work, we speculated therefore that this mutation reduces the steric hindrance during photoisomerization and thereby enhances off-switching. ${ }^{9}$ Spectroscopic investigations into this mutant have furthermore established that the chromophore is deprotonated in the fluorescent on-state. ${ }^{5}$ Since the chromophore is a strong photoacid, ${ }^{5 \mathrm{c}}$ protonation in the excited state is highly unlikely and occurs on the ground state instead. ${ }^{5 b}$ Therefore, our working hypothesis is that the chromophore is deprotonated throughout the excited state dyamics.

In eight out of twelve QM/MM simulations, the chromophore remains planar after photo-excitation, and no decay to the ground state $\left(\mathrm{S}_{0}\right)$ was observed within 50 ps (Figure $\mathrm{S} 11$ in Supporting Information). Instead, the $S_{1} / S_{0}$ energy gap is $292 \pm$ $16 \mathrm{kJmol}^{-1}$ on average and never falls below $100 \mathrm{kJmol}^{-1}$ (Figure Sx, Table S2). Although 50 ps is much shorter than the fluorescence lifetime in this protein, we consider these systems fluorescent, because the planar conformation of the chromophore is apparently stabilized by the protein environment. In the remaining trajectories, a simultaneous rotation of the $\tau$ and $\phi$ torsions was observed within $1 \mathrm{ps}$, bringing the system to a hula-twist conical intersection ${ }^{11}$ with $S_{0}$ (Figure 2). The existence of this intersection and the pathway leading to it were confirmed at the correlated xMCQDPT2 level of theory ${ }^{12}$ (Figure S8 in Supporting Information). In the protein environment the hula-twist photo-isomerization is favored over a single bond flip 
around $\tau$ or $\phi$, because it avoids a steric clash with the histidine side chain that lies stacked on top of the chromophore and is held firmly in place by two ionic hydrogen bonds with glutamate side chains (Figure S12, Supporting Information).
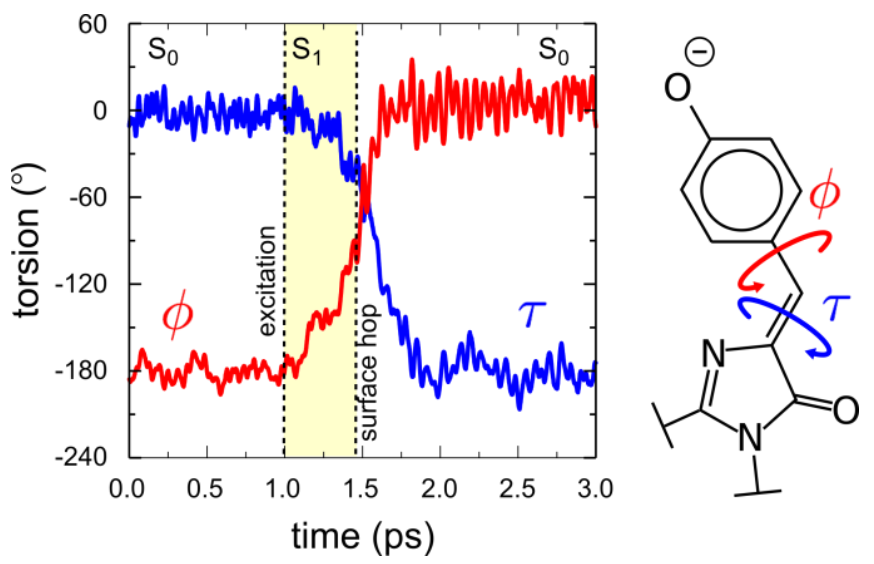

Figure 2. Time evolution of the methine bridge torsions after photo-excitation in a $\mathrm{QM} / \mathrm{MM}$ trajectory initiated from a configuration with a single hydrogen bond to the phenolate moiety (Figure $1 \mathrm{~b}$ ).

After reaching the $S_{1} / S_{0}$ intersection, the system decays to $S_{0}$, restoring the cis conformation of the chromophore in two out of four cases. In the other two cases, the chromophore quickly relaxes into the trans configuration. In line with previous experimental ${ }^{6}$ and computational studies, ${ }^{13}$ we associate the trans chromophore with the non-fluorescent state of Dronpa. Although de-activation of fluorescence also requires protonation, it was shown by Van Thor and co-workers ${ }^{5 b}$ that this occurs after the isomerization and at timescales beyond reach of our QM/MM simulations. We therefore will address the protonation process in the future, using either a classical approach for proton transfer, ${ }^{14}$ a combination of empirical valence bond theory ${ }^{15}$ and the QCFF/PI model, ${ }^{16,17}$ or by means of QM/MM pKa calculations. ${ }^{18}$ Inspection of the starting geometries revealed that rapid photoisomerization occurs only if the phenolate oxygen of the chromophore accepts at most one hydrogen bond from the environment, whereas no deactivation was observed if there are two or more hydrogen bonds. Upon relaxing from the FrankCondon region to the hula-twist conical intersection, about $-0.3 \mathrm{e}$ charge transfers from the phenolate ring onto the imidazolinone ring (Figure Sx in Supporting Information). By stabilizing the negative charge on the phenolate ring, the hydrogen bonds oppose this charge migration and thus introduce a barrier for the photo-isomerization process. Therefore, reducing the number of hydrogen bonds is necessary to remove this barrier and unlock ultra-fast access to the conical intersection (Figure $S x$ in Supporting Information).

The crystal structure of the M159T mutant $^{9}$ suggests three hydrogen bonds: one with Ser142, and two with two crystal waters (Figure 1a). Although this hydrogen bond pattern remains stable on average throughout a 100 ns classical MD simulation, several fluctuations were observed, during which one or two hydrogen bonds are transiently broken (Figures $1 \mathrm{~b}$ and 3). Not only are the internal water molecules exchanging with bulk solvent, also the hydrogen bond with Ser142 breaks and reforms repeatedly (Figure 3). Thus, if, as the QM/MM simulations suggest, two of the hydrogen bonds are required to break in order to activate off-switching, the MM trajectory shows that such events are sufficiently frequent to ensure a significant isomerization quantum yield.

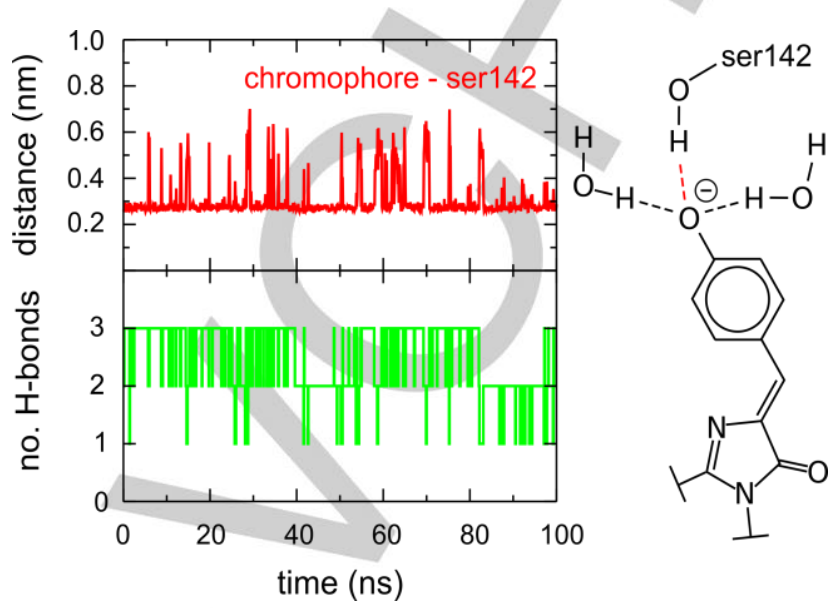

Figure 3. Time evolution of the distance between the hydroxyl group of Ser142 and the chromophore (top) and of the total number of hydrogen bonds that the phenolate moiety forms (bottom) in a classical MD simulation.

The notion that photo-isomerization is only possible during structural fluctuations has to the best of our knowledge not been considered to explain why these proteins are photochromic. Our results therefore not only underscore the necessity to include ground state equilibration in QM/MM simulations of reactive events, ${ }^{15}$ but also reveal a major weakness in focusing solely on the $\mathrm{x}$-ray structure when optimizing the properties of RSFPs.

Furthermore, our findings suggest an alternative strategy to control the switching. The heterogeneity responsible for photochromism may be exploited if the fluorescent and switchable populations can be excited selectively by choosing an appropriate excitation wavelength. Calculations at the xMCQDPT2/cc-pVDZ level ${ }^{12}$ on a larger QM system demonstrate that the absorption wavelength increases with the number of hydrogen bonds (Table S1, Supporting Information). These small differences in excitation energies between the populations suggest that such approach may be possible indeed and could be used not only to verify our predictions, but also to achieve higher resolution in nanoscopy.

In summary, we found that structural heterogeneity in the hydrogen bond network around the chromophore divides the protein ensemble into fluorescent and non-fluorescent populations. To keep the chromophore planar in the excited state and promote fluorescence, two or three hydrogen bonds are required. In contrast, a single hydrogen bond is too weak to prevent isomerization and promotes photo-switching via a hulatwist mechanism instead.

\section{Method Section}


In total $12 \mathrm{QM} / \mathrm{MM}$ surface hopping simulations were initiated from a 100 ns ground state trajectory and run for at most $50 \mathrm{ps}$ in the excited state. In these simulations, the chromophore was described at the $\operatorname{CASSCF}(6,6) / 3-21 \mathrm{G}$ level of theory, ${ }^{16}$ while the remainder was modeled with the Amber03 force field. ${ }^{17}$ Diabatic surface hopping was used to model deactivation at the conical intersection. ${ }^{18}$ Further details of the simulations are given as Supporting Information. Prior to the simulations, the active space was validated by comparing the energies of stationary points to higher levels of theory. In addition, we recomputed the energy profiles along the trajectories at the highly accurate XMCQDPT2/cc-pVDZ level ${ }^{11}$ with a much larger QM system. Details of these and further validation studies, including the effect of including water molecules into the QM region, are described in Supporting Information.

\section{Acknowledgements}

This work was supported by the Academy of Finland. We acknowledge the use of resources of the Moscow State University supercomputing center and CSC - IT center for science, Finland. We thank Prof. Dr. Stefan Jakobs, Prof. Dr. Alexander Nemukhin, Dr. Jasper van Thor and Prof. Dr. Michael Robb for their feedback on the manuscript. We thank Dr. Carl Burmeister for helping us with the analysis of the results.

Keywords: Reversibly switchable fluorescent proteins $•$ photoisomerization $\bullet$ photochromism $\bullet$ structural heterogeneity •

QM/MM

[1] R. Y. Tsien, Annu. Rev. Biochem., 1998, 67, 509-544

[2] E. Betzig et al., Science, 2006, 313, 1642-1645.

[3] M. Hoffmann, C. Eggeling, S. Jakobs, S. W. Hell, Proc. Natl. Acad. Sci. USA, 2005, 102, 17565-17569.

[4] a) R. Ando, H. Mizuno, A. Miyawaki, Science, 2004, 306, 1370-1373; b) S. Habuchi et al., Proc. Natl. Acad. Sci. USA, 2005, 102, 9511-9516.

[5] a) M. M. Warren et al., Nat. Comm., 2013, 4, 1461; b) M. Kaucikas, M. Tros, J. J. van Thor, J. Phys. Chem. B, 2015, 119, 2350-2362; c) Yadav et al., J. Phys. Chem. B, 2015, 119, 2404-2414; d) A. Lukacs et al., J. Phys. Chem. B, 2013, 117, 11954-11959.

[6] M. Andresen et al., Proc. Natl. Acad. Sci. USA, 2007, 104, 13005 13009.

[7] H, Mizuno et al., Proc. Natl. Acad. Sci. USA, 2008, 105, 9227-9232.

[8] T. Brakemann et al., Nat. Biotech., 2011, 29, 942-947.

[9] M. Kaucikas et al., Proteins, 2015, 83, 397-402.

[10] A. C. Stiel et al., Biochem. J., 2007, 402, 35-42.

[11] a) R. S. H. Liu, Acc. Chem. Res. 2011, 34, 555-562; b) R. S. H. Liu, G. S. Hammond, Proc. Natl. Acad. Sci. U.S.A., 2000, 97, 11153-11158.

[12] A. A. Granovsky, J. Chem. Phys., 2011, 134, 214113.

[13] X. Li et al., J. Phys. Chem. Lett., 2010, 1, 3328-3333.

[14] M. G. Wolf, G. Groenhof, J. Comp. Chem., 2014, 35, 657-671.

[15] A. Warshel, R. Weiss, J. Am. Chem. Soc., 1980, 102, 6218-6226.

[16] a) A. Warshel, Isr. J. Chem. 1973, 11, 709-717; b) A. Warshel, A Lappicirella, J. Am. Chem. Soc. 1981, 103, 4664-4673; c) N. V. Plotnikov, PhD Thesis, Univ. of Southern California, 2013, 95-100.

[17] A. Braun-Sand et al., Biochim. Biophys. Acta, Bioenerg., 2008, 1777, 441-452.

[18] S. C. L. Kamerlin, M. Haranczyk, A. Warshel, J. Phys. Chem. B, 2009 113, 1253-1272.

[19] M. Klähn, S. Braun-Sand, E. Rosta, A. Warshel, J. Phys. Chem. B, 2005, 109, 15645-15650.

[20] P. E. M. Siegbahn, J. Almlof, A. Heiberg, B. O. Roos, J. Chem. Phys. 1981, 74, 2384-2396.

[21] Y. Duan et al., J. Comp. Chem., 2003, 24, 1999-2012.

[22] M. Boggio-Pasqua, C. F. Burmeister, M. A. Robb, G. Groenhof, Phys. Chem. Chem. Phys., 2012, 14, 7912-7928. 


\section{Entry for the Table of Contents}

\section{COMMUNICATION}

Structural heterogeneity divides the ground state ensemble of reversibly photo-switchable fluorescent proteins into two populations, of which the major one fluoresces upon photon absorption, whereas the minor population deactivates into a dark non-fluorescent state.
Dmitry Morozov, Gerrit Groenhof*

Page No. - Page No.

Hydrogen bond fluctuations control photochromism in a reversibly photo-

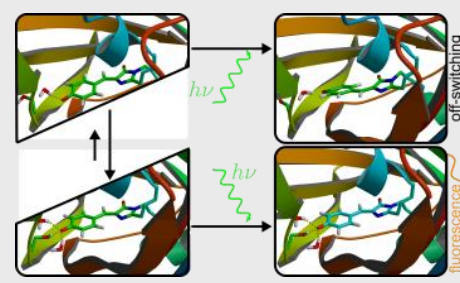

switchable fluorescent protein 